\title{
Insights into local shockwave behavior and thermodynamics in granular materials from tomography-initialized mesoscale simulations
}

Cite as: J. Appl. Phys. 125, 015902 (2019); https://doi.org/10.1063/1.5048591

Submitted: 16 July 2018 . Accepted: 01 December 2018 . Published Online: 03 January 2019

M. E. Rutherford, J. G. Derrick, D. J. Chapman, G. S. Collins, and D. E. Eakins (D)

\section{COLLECTIONS}

This paper was selected as Featured
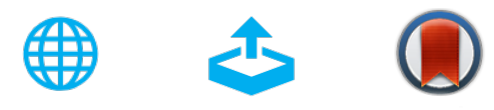

\section{ARTICLES YOU MAY BE INTERESTED IN}

Phonon properties and thermal conductivity from first principles, lattice dynamics, and the Boltzmann transport equation

Journal of Applied Physics 125, 011101 (2019); https://doi.org/10.1063/1.5064602

Dynamic evolution of microstructure during laser shock loading and spall failure of single crystal Al at the atomic scales

Journal of Applied Physics 124, 205901 (2018); https://doi.org/10.1063/1.5051618

In situ X-ray imaging of heterogeneity in dynamic compaction of granular media Journal of Applied Physics 125, 025902 (2019); https://doi.org/10.1063/1.5057713

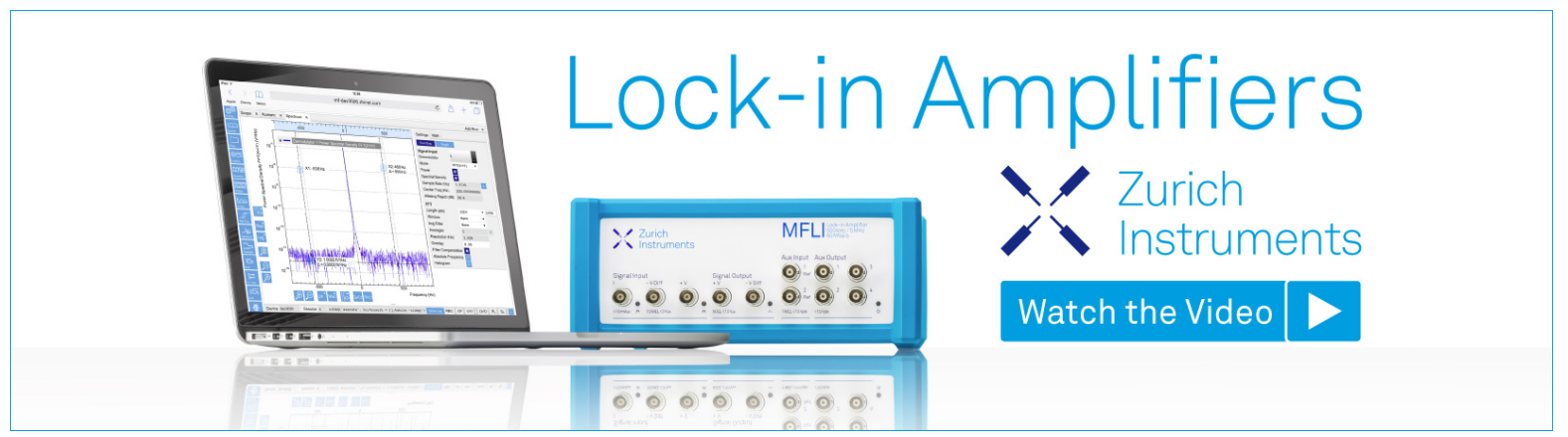




\title{
Insights into local shockwave behavior and thermodynamics in granular materials from tomography-initialized mesoscale simulations
}

\author{
Cite as: J. Appl. Phys. 125, 015902 (2019); doi: 10.1063/1.5048591 \\ Submitted: 16 July 2018 . Accepted: 1 December 2018 . \\ Published Online: 3 January 2019
}

M. E. Rutherford, ${ }^{1, a)}$ J. G. Derrick, ${ }^{2}$ D. J. Chapman, ${ }^{1, b)}$ G. S. Collins, ${ }^{2}$ and D. E. Eakins ${ }^{1, b)}$ (D)

\author{
AFFILIATIONS \\ ${ }^{7}$ Solid Mechanics and Materials Engineering, University of Oxford, Oxford, United Kingdom \\ ${ }^{2}$ Department of Earth Science and Engineering, Imperial College London, London, United Kingdom \\ a) michael.rutherford@eng.ox.ac.uk \\ b) Also at Institute of Shock Physics, Imperial College London, London, UK.
}

\begin{abstract}
Interpreting and tailoring the dynamic mechanical response of granular systems relies upon understanding how the initial arrangement of grains influences the compaction kinetics and thermodynamics. In this article, the influence of initial granular arrangement on the dynamic compaction response of a bimodal powder system (soda-lime distributed throughout a porous, fused silica matrix) was investigated through continuum-level and mesoscale simulations incorporating real, as-tested microstructures measured with X-ray tomography. By accounting for heterogeneities in the real powder composition, continuum-level simulations were brought into significantly better agreement with previously reported experimental data. Mesoscale simulations reproduced much of the previously unexplained experimental data scatter, gave further evidence of low-impedance mixture components dominating shock velocity dispersion, and crucially predicted the unexpectedly high velocities observed experimentally during the early stages of compaction. Moreover, only when the real microstructure was accounted for did simulations predict that small fractions of the fused silica matrix material would be driven into the $\beta$-quartz region of phase space. These results suggest that using real microstructures in mesoscale simulations is a critical step in understanding the full range of shock states achieved during dynamic granular compaction and interpreting solid phase distributions found in real planetary bodies.
\end{abstract}

Published under license by AIP Publishing. https://doi.org/10.1063/1.5048591

\section{INTRODUCTION}

The dynamic compaction of granular systems is a complex process occurring in a number of scenarios including materials by design, ${ }^{1,2}$ impact-driven planetary body formation and asteroid defeat, ${ }^{3,4}$ explosive mining, ${ }^{5}$ and blast mitigation. ${ }^{6}$ The extensive number of initial physical parameters that are available to optimize when designing next-generation granular systems for these environments (e.g., initial: grain size, grain morphology, porosity, packing arrangement, grain surface roughness, and others) simultaneously make the dynamic mechanical response of granular systems tremendously adjustable but also challenging to model. ${ }^{7,8}$ Accurately predicting the dynamic behavior and thermodynamic conditions in granular systems under impact loading is further complicated when the system is composed of multiple components as is the case in plastic-bonded explosives, astrophysical systems, and composites. ${ }^{3,9-12}$

While the literature details numerous focused studies of the effect of a single initial parameter such as grain size on, for example, shockwave rise-times and crush-strengths, ${ }^{13-16}$ there have been relatively few studies considering the influence of initial granular packing (the arrangement of the grains in the sample volume) on the dynamic compaction response. This is problematic for explaining existing experimental results and designing future materials with predictable performance as configuration-dependent phenomena such as stress-bridging and hot-spot formation are often reasoned to dominate dynamic granular compaction. ${ }^{7,8,17,18}$ Eakins et al. imported real powder microstructures into the CTH hydrocode via processing electron microscopy images recorded on 
polymer-cast, sectioned samples of a $\mathrm{Ni}+\mathrm{Al}$ powder volume. Significant strain, pressure, and temperature variations were predicted with, for example, twice the strain seen in $\mathrm{Al}$ grains in comparison with Ni grains. Moreover, in spherically grained mixtures, pressure deviations of $10 \mathrm{GPa}$ about the mean were observed, whereas only a deviation of $1 \mathrm{GPa}$ was observed in the flake mixtures. ${ }^{15}$ While these microscopybased studies have seen many successes, they necessitated destruction of the powder volumes and thus could not directly compare the same measured microstructure through experiment and modeling.

In contrast to the microscopy methods employed by Eakins and Thadhani ${ }^{15}$ and Singh et al., ${ }^{19,20}$ X-ray tomography does not require granular samples to be cast in a polymer and sectioned prior to study. A notable use of X-ray tomography to provide realistic mesoscale shock simulation geometries is a detailed investigation of heterogeneity in aluminumpolyvinylidene fluoride (Al-PVDF) foams. ${ }^{17}$ This computational investigation, which reproduced experimental measurements of particle velocity in a copper witness plate impacted by the Al-PVDF foam, revealed numerous statistical insights: a Gaussian distribution of stress in the system, differences of $200 \mathrm{~K}$ between the $\mathrm{Al}$ and PVDF components, and $0.1 \%$ of the PVDF experiencing hotspots of greater than $1000 \mathrm{~K}$. The influence of the simulated microstructure on the dynamic compaction response was further investigated by comparing three different initializations: a simulation microstructure initialized from tomography measurements of the bulk foam, a second simulation microstructure initialized from tomography measurements of only the leading $0.1 \mathrm{~mm}$ portion of the sample (which was thought to dominate witness plate observations), and finally an artificially generated microstructure with knowledge of only the bulk component volume fractions. Simulations performed with these three microstructures all showed variation in the most probable stress, demonstrating that even small changes in component volume fractions and how the components are arranged in the sample volume markedly affect the macroscale response. ${ }^{17}$ These statistical observations have been increasingly supported by a growing body of experimental and computational work on brittle granular materials that report particle velocity distributions with widths directly proportional to grain size ${ }^{11}$ the need to account for matrix material phase-changes in multi-component systems such as water-saturated sand, ${ }^{12}$ and observations of shock velocity distributions. $^{18}$

This work uses continuum-level and mesoscale simulations to further investigate the dynamic compaction response of a bimodal powder system, for which experimental compaction measurements were previously reported by Rutherford et al. $^{18}$ The experiments are briefly reviewed here to provide context for this article. The studied powder system was designed to mimic a precursor chondritic meteorite material and therefore contained $70 \%$ by volume porous fused silica (FS) and $30 \%$ by volume soda-lime (SL) microspheres. The average density of the fused silica was $27 \%$ that of the solid (hereby referred to as $27 \%$ theoretical maximum density or TMD). Mixtures were prepared with one of the two soda-lime microsphere sizes and referred to as large grain $(425 \mu \mathrm{m}$ mean diameter) and small grain $(196 \mu$ m mean diameter) mixtures. Shock front and interface motion in the powder bed during dynamic compaction were recorded by two high-resolution X-ray radiographs typically spaced $2 \mu \mathrm{s}$ apart. The first and second radiographs recorded in each experiment are referred to as Frame 1 (F1) and Frame 2 (F2), respectively. Spatially resolved, time-averaged measurements of local shock and particle velocity over the shock transit times were extracted from the radiographs. Particle velocity was taken to be equal to the velocity between the interface of the driver and powder. Spatially resolved shock and particle velocities in each radiograph were spatially averaged over a centered, vertical region equal to 10 mean soda-lime microsphere diameters and are therefore referred to as on-axis velocities. This averaging was performed to facilitate a comparison with computation models and to limit the effects of lateral release. Within the on-axis regions, a Gaussian distribution of velocities was observed from which standard deviations were taken as the measure of error in the average velocity, which greatly exceeded any errors in the experimental timeline. Reasonable agreement of a continuum-level, mixture Hugoniot with the average shock, and particle velocities were reported. However, anomalously high shock and particle velocities measured from the earliest radiographs in the experimental dataset were not predicted by the continuum-level model and could not be explained by timing uncertainties. These outlier velocities were therefore thought to be due to highly inhomogeneous soda-lime distributions throughout the sample volumes resulting in processes such as stress bridging. Calculations using an X-ray absorption model showed that precursor waves would have produced too small an intensity change with respect to the noise in the radiographs to be detected, further details of which are presented in the supplementary material. ${ }^{21}$ Therefore, the densification waves observed were treated as shock waves and referred to as such even though they were strictly speaking compaction waves relying upon partial granular rearrangement.

In this article, X-ray tomography data collected prior to the previously reported dynamic experiments are presented. Measurements of the real experimental microstructure are incorporated into continuum-level and mesoscale models with the intention of predicting a wider range of shock and particle velocities over those previously obtained without explicit knowledge of the experimental microstructure. These simulations also provide insight into the local distribution of velocity, temperature, and pressure in the shocked powder. Details of the tomography measurements, analysis, and extraction of component volume fractions are presented in Sec. II. Section III presents a range of continuumlevel predictions after which Sec. IV provides further insight into the local conditions within the shocked powder through mesoscale simulations. A key goal of this work is to understand the full range of velocity-temperature-pressure states produced in a dynamically compacted granular system, including those at early times long before a steady wave has been formed. 


\section{TOMOGRAPHY RESULTS}

X-ray tomography was conducted on Beamline ID19 at the European Synchrotron Radiation Facility (ESRF, France) with a single-harmonic undulator operating at $26 \mathrm{keV}$. A total of 1500 projections were recorded over $180^{\circ}$ on a FReLoN2k camera with an effective pixel size of $6.44 \mu \mathrm{m}$. A sample-scintillator (YAG:Ce) distance of $3 \mathrm{~m}$ introduced phase-contrast into the radiographs. At this pixel size, it was not possible to resolve grains in the matrix material but the soda-lime microspheres were well-resolved. Images were reconstructed using a filtered back projection algorithm and the method of Lyckegaard et al. to pre-correct ring artifacts, both built into the ID19 tomography user pipeline. ${ }^{22}$ To quantify the area fraction of soda-lime microspheres in each reconstructed slice, and ultimately the heterogeneity of the mixture, the images were thresholded using Otsu's method ${ }^{23}$ after masking the gray levels associated with the aluminum powder cell. Accurate segmentation of the two phases required singledistance phase-retrieval using Paganin et al.'s algorithm ${ }^{24}$ for which a ratio of the refractive index decrement to absorption index $\left(\frac{\delta}{\beta}\right)$ of 100 provided adequate segmentation.

Figures 1(a) and 1(b) show, respectively, static and dynamic radiographs recorded on a large grained mixture shot (Shot 6, Table I). This shot was selected because unexpectedly high F1 particle and shockwave velocities of $878 \pm 54 \mathrm{~ms}^{-1}$ and 3359 $\pm 211 \mathrm{~ms}^{-1}$ were recorded during its dynamic compaction, respectively. It was previously concluded that this high shock speed was a result of waves racing through the soda-lime dense region near the driver interface ${ }^{18}$ [judged qualitatively from the decreased transmission in Fig. 1(a)]. To extend this hypothesis quantitatively, the measured soda-lime area fractions as a function of longitudinal position across the whole cell diameter (total) and across the central, on-axis column (on-axis) as a function of longitudinal position in Figs. 1(c) and $1(d)$, respectively. The composition of the powder was notably more heterogeneous in the on-axis column with both the total and on-axis measurements showing significant (e.g., up to $\sim 60 \%$ ) excursions away from their mean area fractions.

Table I summarises the 7 experimental datasets (shots) analyzed in this article, including the impact conditions and time of F1 and F2 after the shock entered the powder bed (shock time). Table I also shows the measured soda-lime volume fractions for three defined volumes in the powder bed: that for the entire sample, $\mathrm{V}_{\mathrm{SL} \text {,Total }}$; that for the volume spanned by the static driver position and the shockwave position measured in $\mathrm{F} 1, \mathrm{~V}_{\mathrm{SL}, \mathrm{F} 1}$; and that for the volume spanned by the static driver position and the shockwave position measured in $\mathrm{F} 2, \mathrm{~V}_{\mathrm{SL}, \mathrm{F} 2}$. For each sample, the total volume fraction of soda-lime was within the sample preparation uncertainty bounds $( \pm 4 \%)$ of the desired $30 \%$. In contrast, the volume traversed by the shockwave front in F1 and F2 spanned $20-48 \%$ across the seven shots, showing that the shockwave encountered powder compositions significantly different to the intended bulk composition. Table I also shows the total $\left(\mathrm{V}_{\mathrm{SL}, \mathrm{Total}, \text { on-axis }}\right), \mathrm{F} 1\left(\mathrm{~V}_{\mathrm{SL}, \mathrm{F} 2, \text { on-axis }}\right)$, and F2 $\left(\mathrm{V}_{\mathrm{SL}, \mathrm{F} 2, \text { on-axis }}\right)$ volume fractions in the on-axis volumes, which exhibited soda-lime volume fractions of $26-51 \%$. For brevity, polycarbonate is referred to as PC in Table I.

\section{CONTINUUM-LEVEL PREDICTIONS}

Continuum-level calculations were first performed to investigate the origin of the highest experimental velocities and data scatter. To be consistent with previous works, the mixture theory proposed by Batsanov ${ }^{25}$ was used to calculate the Hugoniot for the precursor chondrite mixtures (hereby referred to as the mixture Hugoniot). This approach required Hugoniots for the soda-lime and porous, fused silica components. For a given shock stress, the mixture particle velocity was calculated as the root-mean-square of the component particle velocities, averaged by their mass fractions. This is shown as follows:

$$
\mathrm{U}_{p, \text { mix }}(\sigma)=\sqrt{\mu_{\mathrm{SL}} \cdot u_{p, \mathrm{SL}}(\sigma)^{2}+\mu_{\mathrm{FS}} \cdot u_{p, \mathrm{FS}}(\sigma)^{2}},
$$

where $U_{p, \text { mix }}(\sigma)$ is the mixture particle velocity at a stress $\sigma$, $u_{p}(\sigma)$ is the particle velocity of a component at stress $\sigma$, and $\mu$ is the mass fraction of the component. The subscript SL refers to solid soda-lime and FS to porous fused silica. The associated shock velocity for the mixture was calculated using

$$
\mathrm{U}_{\mathrm{s}, \text { mix }}(\sigma)=\frac{\sigma}{\left[\rho_{0, \text { mix }} \cdot u_{p, \text { mix }}(\sigma)\right]},
$$

where $U_{s, \text { mix }}(\sigma)$ is the mixture shock velocity at a stress $\sigma$. Equation (3) defines the volume-weighted initial density

$$
\rho_{0, \text { mix }}=v_{\mathrm{SL}} \cdot \rho_{0, \mathrm{SL}}+v_{\mathrm{FS}} \cdot \rho_{0, \mathrm{FS}},
$$

where $\rho_{0, \text { mix }}$ is the mixture initial density and $v$ is the initial volume fraction of the mixture component.

The soda-lime volume fractions obtained through X-ray tomography were included in these calculations to produce a unique mixture Hugoniot for each of the 7 shots. The component Hugoniots for soda-lime and fused silica were calculated using a Mie-Grüneisen equation of state. Grüneisen constants of 0.14 and 0.036 were used for soda-lime and fused silica, respectively. ${ }^{26,27}$

The influence of initial porosity in the fused silica Hugoniot was accounted for using the $\epsilon-\alpha$ model ${ }^{28,29}$ The $\epsilon-\alpha$ parameters: $\alpha_{x}$ (distension at the transition from exponential to power-law compaction regimes), $\kappa$ (compaction rate parameter), and $\chi$ (ratio of porous sound speed to that of the solid) were determined by comparison with plate-impact data measured previously for 35\% TMD fused silica. ${ }^{30}$ Table II shows the $\epsilon-\alpha$ parameters used in the calculation. The Druker-Prager strength model ${ }^{31}$ was used to model the strength of porous fused silica. Table III shows the strength model parameters used in the calculation. The calculated Hugoniot for soda-lime was in good agreement with that reported in the literature. ${ }^{32}$ Soda-lime was treated with an elastic-perfectly plastic strength model and a yield stress of $1 \mathrm{GPa}$.

Figure 2 shows the velocity data recorded on F1 in shock velocity-particle velocity space with an expanded range of 


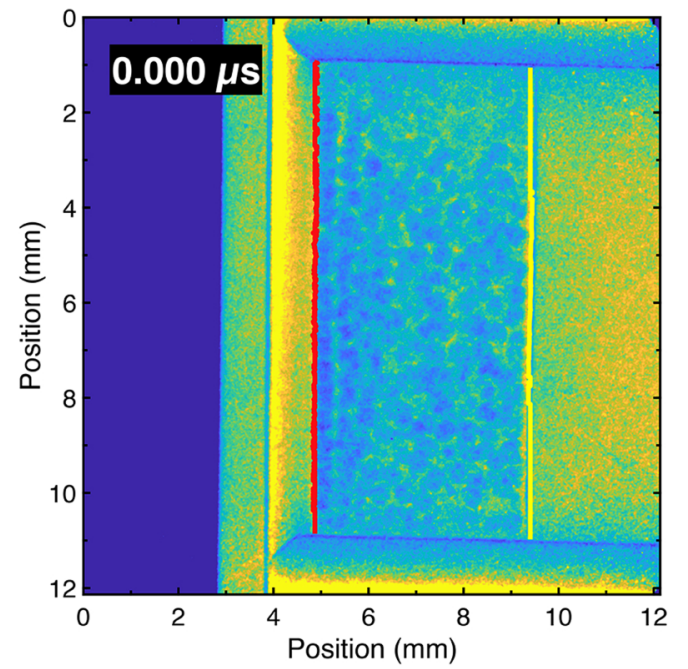

(a)

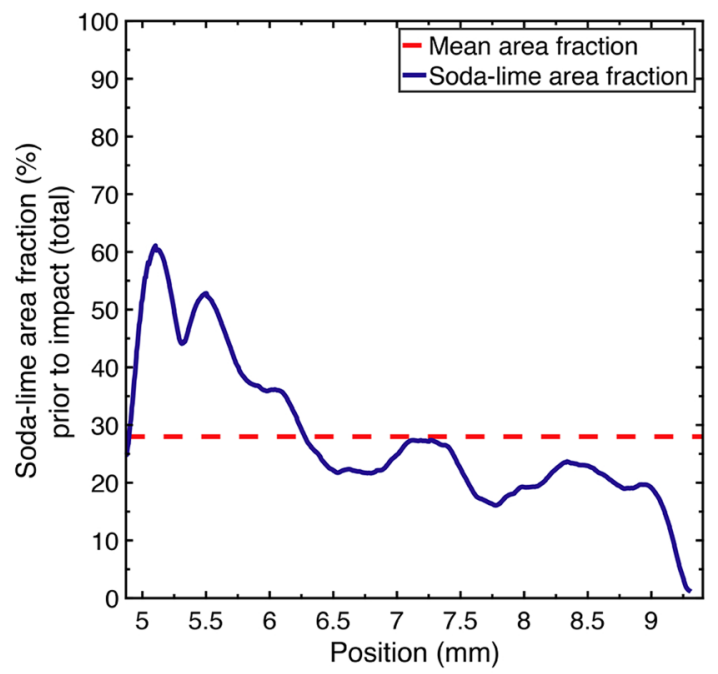

(c)

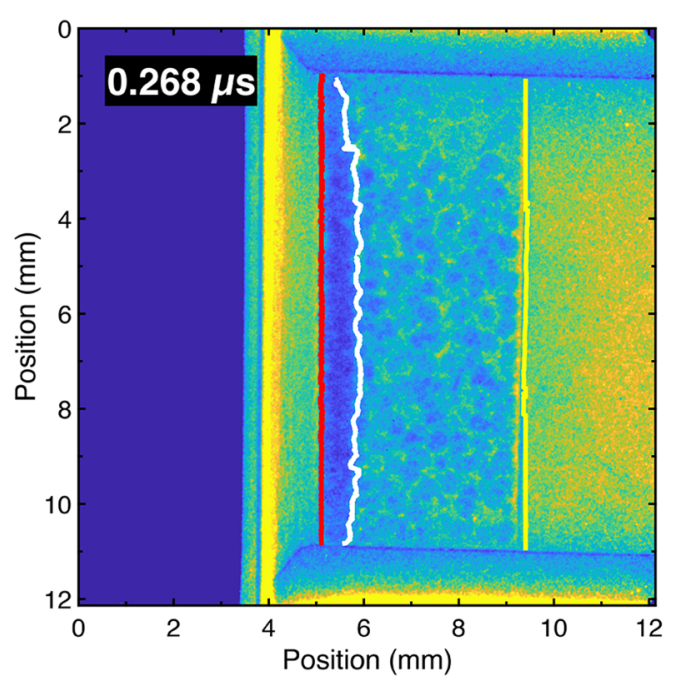

(b)

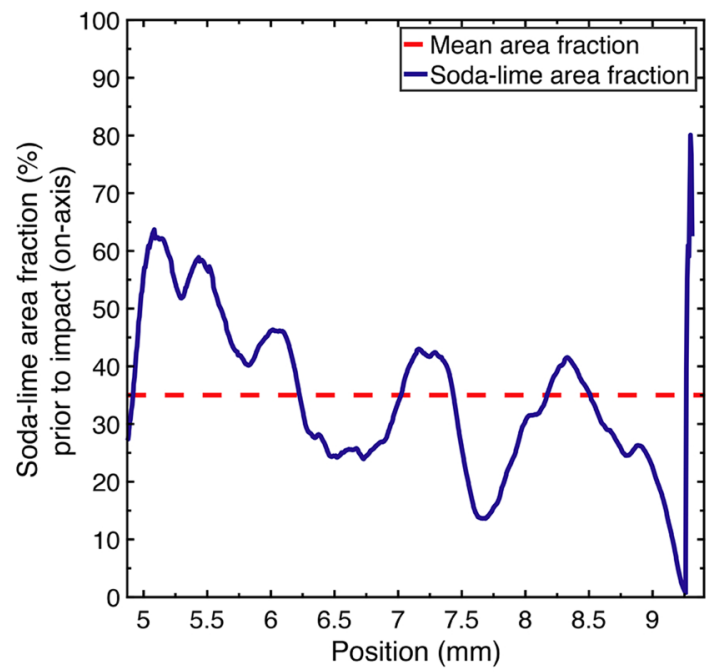

(d)

FIG. 1. Example static and dynamic radiographs from which velocity measurements were made in Rutherford et al. ${ }^{18}$ alongside soda-lime area fractions measured from X-ray tomography. (a) A static radiograph recorded prior to impact on a large grained mixture (Shot 6, Table I). The first and second radiographs recorded in each experiment are referred to as Frame 1 (F1) and Frame 2 (F2), respectively. Red and yellow lines mark the measured interfaces between the driver and powder, and powder and window, respectively. (b) The dynamic radiograph recorded in F1 on the same shot, $0.268 \pm 0.003 \mu$ s after the shockwave entered the powder bed. A white line marks the measured shock front position. (c) and (d) Measured total and on-axis soda-lime area fraction as a function of longitudinal position along the powder bed determined from tomography, respectively. The position axes in (c) and (d) are linked to those shown in (a) and (b) to facilitate easier comparison.

predicted states at the continuum-level. The three outlier data are identified by the numbers 1,2 , and 3 (white text on black circles). The newly predicted states were calculated from the full range of measured, on-axis, soda-lime volume fractions of $26-51 \%$. For this range of mixture Hugoniots, it was assumed that the fused silica density was equal to the average experimental density of $27 \%$ TMD. To model the fused silica at 27\% TMD only the initial density was adjusted from the previously determined parameters in Table II. To aid visual comparison of the experimental data with the calculated predictions, each F1 data point is surrounded by concentric rectangles, indicating up to 3 standard deviations of the measured on-axis velocity distributions. The expanded range of continuum-level states now intersects 3 of the $7 \mathrm{~F} 1$ data directly and 5 within three standard deviations. This agreement is a significant improvement over the earlier 
TABLE I. Measured soda-lime microsphere volume fractions for chondrite precursor mixture shots.

\begin{tabular}{|c|c|c|c|c|c|c|c|c|c|c|c|c|}
\hline $\begin{array}{l}\text { Shot } \\
\text { no. }\end{array}$ & $\begin{array}{l}\text { Grain } \\
\text { size }\end{array}$ & $\begin{array}{c}\text { Flyer } \\
\text { material }\end{array}$ & $\begin{array}{c}\text { Driver } \\
\text { material }\end{array}$ & $\begin{array}{l}\text { Impact } \\
\text { velocity } \\
\left(\mathrm{ms}^{-1}\right)\end{array}$ & $\begin{array}{c}\text { F1 shock time } \\
(\mu \mathrm{s})\end{array}$ & $\begin{array}{c}\text { F2 shock time } \\
\text { (us) }\end{array}$ & $\begin{array}{c}V_{S L} \\
\text { total } \\
(\%)\end{array}$ & $\begin{array}{c}V_{S L} \\
F 1 \\
(\%)\end{array}$ & $\begin{array}{l}V_{S L} \\
F 2 \\
(\%)\end{array}$ & $\begin{array}{c}V_{S L} \text { total } \\
\text { on-axis }(\%)\end{array}$ & $\begin{array}{c}V_{S L} F 1 \\
\text { on-axis } \\
(\%)\end{array}$ & $\begin{array}{c}V_{S L} F 2 \\
\text { on-axis } \\
(\%)\end{array}$ \\
\hline 1 & Small & PC & PC & $628 \pm 2$ & $1.236 \pm 0.008$ & $3.353 \pm 0.011$ & 32 & 22 & 32 & 41 & 26 & 43 \\
\hline 2 & Small & PC & PC & $633 \pm 2$ & $2.254 \pm 0.011$ & $4.370 \pm 0.011$ & 29 & 23 & 29 & 40 & 45 & 40 \\
\hline 3 & Large & PC & PC & $627 \pm 2$ & $1.341 \pm 0.008$ & $3.457 \pm 0.011$ & 31 & 24 & 31 & 25 & 33 & 27 \\
\hline 4 & Large & PC & PC & $633 \pm 2$ & $2.177 \pm 0.011$ & $4.295 \pm 0.011$ & 33 & 31 & 33 & 38 & 38 & 38 \\
\hline 5 & Large & $\mathrm{Cu}$ & PC & $598 \pm 1$ & $0.909 \pm 0.006$ & $3.026 \pm 0.011$ & 29 & 29 & 29 & 34 & 33 & 34 \\
\hline 6 & Large & $\mathrm{Cu}$ & $P C$ & $598 \pm 1$ & $0.268 \pm 0.003$ & $2.385 \pm 0.010$ & 28 & 48 & 29 & 35 & 51 & 36 \\
\hline 7 & Small & $\mathrm{Cu}$ & PC & $595 \pm 1$ & $0.820 \pm 0.006$ & $2.937 \pm 0.011$ & 27 & 30 & 25 & 39 & 38 & 38 \\
\hline
\end{tabular}

predictions of Rutherford et al., which only matched 1 datum directly and 4 within three standard deviations. ${ }^{18}$ The improved agreement with experimental velocity data confirms the usefulness of non-destructively characterizing the sodalime packing within the powder volume prior to dynamic compaction. However, the full range of measured velocity data was not captured by the continuum-level predictions.

To explore further the soda-lime volume fractions required to reproduce the unexpectedly high velocity outlier data at the continuum level, Fig. 3 shows the same experimental data with continuum-level Hugoniots calculated for soda-lime volume fractions spanning now $0-100 \%$. It was again assumed that the fused silica density was equal to the average experimental density of $27 \%$ TMD. To reach the mean velocities of the three outlier data, soda-lime volume fractions of $60-70 \%$ (outliers 1 and 2) and $90-100 \%$ (outlier 3) are required. These soda-lime volume fractions are significantly higher than the mean volume fractions measured by tomography in the volumes spanned by the shockwave. The shortcomings of the continuum-level model in predicting the outlier velocities suggest that it is crucial to incorporate mesoscale effects such as spatially varying volume fractions and stress-bridging into velocity predictions. The possibility that favorable transmission of stress through chondrule-chondrule contacts could have produced the anomalously high velocities observed is consistent with the micro-structural characterization revealing majority soda-lime regions, and measurable motion in the radiographs being dominated by the soda-lime microspheres.

\section{MESOSCALE PREDICTIONS}

\section{A. Mesoscale simulations}

This section investigates the differences between mesoscale simulations initialized using an idealized microstructure and simulations initialized from tomography measurements of the real experimental microstructure. The primary goal of this section is to highlight the importance of using experimental microstructures in simulations and to understand the shortcomings of idealized simulation microstructures, which are generated with only knowledge of the component volume fractions. Mesoscale simulations were performed using the 2D shock physics code iSALE. 28,33

Firstly, four simulations, one to cover each experimental grain size and impact condition, were first performed with a powder bed in which the location of the soda-lime microspheres throughout the porous silica volume was random. These simulations are therefore referred to as idealized simulations. The powder beds were $30 \%$ by area, single size sodalime circles with the remainder of the area occupied by $27 \%$ TMD fused silica.

Secondly, simulations were performed using powder beds and grain locations extracted directly from tomography data recorded on the powder mixtures prior to impact. These simulations are referred to as tomography-initialized simulations. To do so, a longitudinal slice was taken through the reconstructed volume and the powder bed cropped from it. Thresholding was then performed to segment the soda-lime sphere positions, and watershed segmentation was utilised to ensure adjacent grains were not artificially welded in the simulations. It was assumed that all the porous fused silica matrix material in a tomography-generated microstructure has the same density because it was not possible to measure local variations in the silica density with tomography. For a given shot, this density was calculated with knowledge of the sodalime volume fraction, component masses, and sample dimensions and spanned 25-29\% TMD across the 7 shots. Simulations considered only the central column (sabot, driver, powder cell, and window) of the experimental geometry due to limited computational time. The simulation cell

TABLE II. $\epsilon-\alpha$ compaction model parameters for porous fused silica.

\begin{tabular}{lcccc}
\hline \hline $\begin{array}{l}\text { Initial density } \\
\left(\mathrm{g} \mathrm{cm}^{-3}\right)\end{array}$ & $\begin{array}{c}\text { Initial } \\
\text { porosity } \\
\boldsymbol{\alpha}_{0}\end{array}$ & $\begin{array}{c}\text { Transition } \\
\text { distension } \\
\boldsymbol{\alpha}_{\boldsymbol{x}}\end{array}$ & $\begin{array}{c}\text { Exponential } \\
\text { coefficient } \\
\boldsymbol{\kappa}\end{array}$ & $\begin{array}{c}\text { Sound } \\
\text { speed ratio } \\
\boldsymbol{\chi}\end{array}$ \\
\hline 2.20 & 3.85 & 1.29 & 0.8 & 0.33 \\
\hline \hline
\end{tabular}

TABLE III. Drucker-Prager strength model parameters for porous fused silica.

\begin{tabular}{lccc}
\hline \hline $\begin{array}{l}\text { Cohesion } \\
(\mathrm{kPa})\end{array}$ & $\begin{array}{c}\text { Coefficient of internal } \\
\text { friction }\end{array}$ & $\begin{array}{c}\text { Limiting strength } \\
\text { (MPa) }\end{array}$ & $\begin{array}{c}\text { Poisson } \\
\text { ratio }\end{array}$ \\
\hline 10 & 0.7 & 100 & 0.3 \\
\hline \hline
\end{tabular}




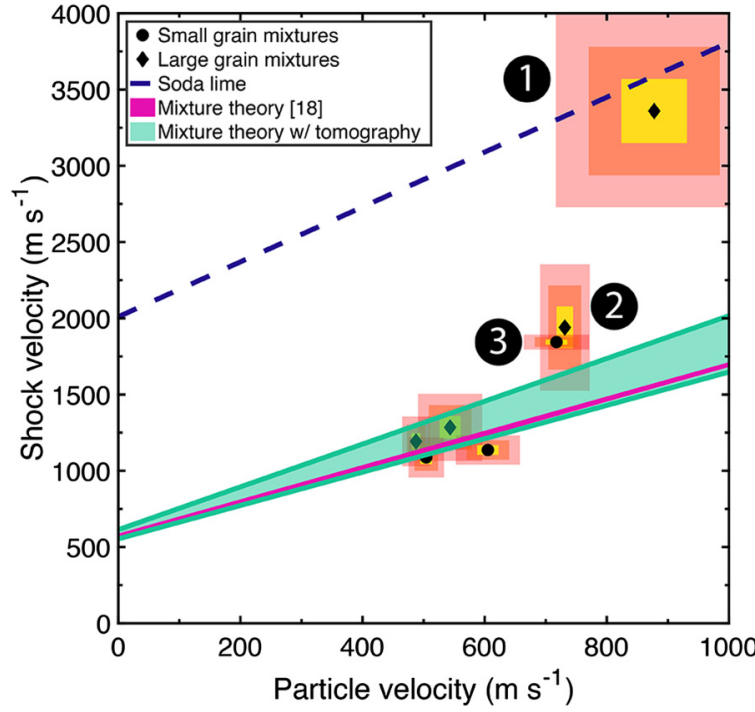

FIG. 2. Comparison of velocity data recorded on F1 during the dynamic compaction of chondrite precursor mixtures with continuum-level predictions calculated using the measured soda-lime volume fractions. Each datum is shown with 1 (yellow), 2 (orange), and 3 (red) concentric rectangular bands representing 1 , 2 , and 3 standard deviations of the measured on-axis distributions. A solid pink band shows the range of predicted velocity states from Rutherford et al. ${ }^{18} \mathrm{~A}$ green band shows the increased range of predicted velocity states in this work calculated using soda-lime volume fractions measured with X-ray tomography. The solid soda-lime Hugoniot is shown by a dark, dashed blue line. The three outlier data are identified by the numbers 1,2 , and 3 .

size was equal to the tomography voxel dimension, $6.44 \mu \mathrm{m}$. To investigate whether the tomography-initialized mesoscale simulation results were sensitive to the choice of powder bed cropped from the tomography volume, three tomography-generated input geometries-separated by $120^{\circ}$ about the powder cell's axis of symmetry-were simulated for each shot. From inspection of the longitudinal velocity maps in the simulations, a threshold particle velocity of $50 \mathrm{~ms}^{-1}$ was chosen to define the shock front position in both the soda-lime and porous silica components. This choice of shock front definition delivered an acceptably similar result to previous methods used in iSALE, which defined the threshold particle velocity for the shock front as $\frac{1}{3}$ impact velocity. ${ }^{34}$ As was the case for the continuum-level simulations, only the initial porosity in the $\epsilon-\alpha$ compaction model was adjusted when modeling these silica densities of 25-29\% TMD. This was because no experimental data were available to optimize the model parameters at 25-29\% TMD. The scope of this article was to investigate the effect of granular arrangement on the compaction process and not refine the fused silica equation of state, which was kept otherwise consistent in all simulations.

It is important to emphasize and clarify the multitude of data extracted from the mesoscale simulations at this stage. Simulation results were exported with a timestep of $50 \mathrm{~ns}$. At

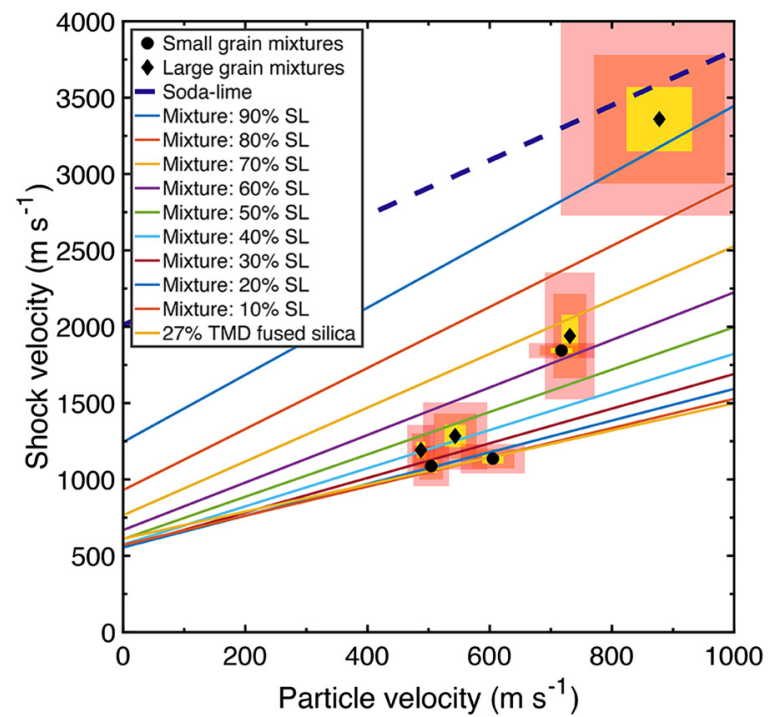

FIG. 3. Comparison of velocity data recorded on F1 during the dynamic compaction of chondrite precursor mixtures with continuum-level predictions calculated using soda-lime volume fractions of $0 \%-100 \%$. Error bars are shown as before in Fig. 2. Continuum-level, mixture Hugoniots with varying volume fractions of soda-lime are shown as colored lines. These Hugoniots are bounded by the 27\% TMD fused silica Hugoniot and the solid, soda-lime Hugoniot. Mixture Hugoniots contain the stated volume fraction of soda-lime, mixed with 27\% TMD fused silica.

each timestep, a shock position and a driver position were calculated. These positions were taken as local measurements of the in-powder, heterogeneous shock, and particle position. By taking the timestep-to-timestep shock and driver transit distance and dividing through by the timestep duration, a temporally resolved measure of shock and driver speed was calculated and shall be referred to as the local measurement from now on. In contrast, temporally averaged shock and driver velocities were also calculated by considering, at a given timestep, the distance traveled by the shock and driver positions relative to the static driver position and dividing through by the total transit time. These velocities are hereby referred to as time-averaged, which are analogous to experimental radiography measurements in which distinct observations are made relative to a known reference position. Again, the particle velocity in the powder mixture was taken equal to the driver velocity.

\section{B. Initial microstructure}

Figure 4 shows the effect of the sample microstructure on the local variations in simulated shock and particle velocity, and how this differs depending on whether a randomly initialized or tomography-initialized microstructure is used. The experimental microstructure used here is one in which a $\mathrm{Cu}$ flyer struck a polycarbonate-driver powder cell filled with a large grain mixture at $598 \mathrm{~ms}^{-1}$, referred to as Shot 5 in 

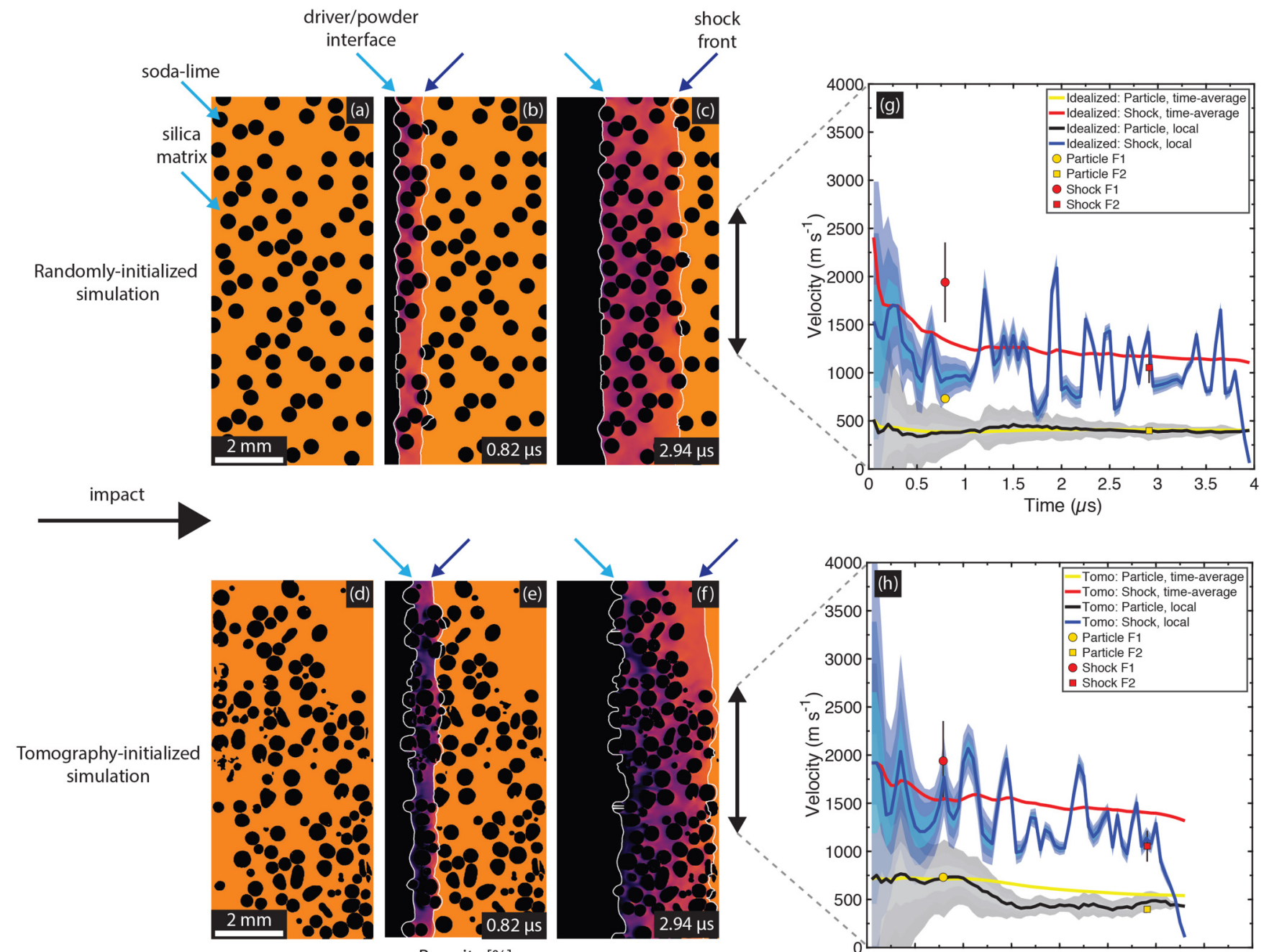

Porosity [\%]

\begin{tabular}{llllll}
100 & 80 & 60 & 40 & 20 & 0 \\
\hline
\end{tabular}

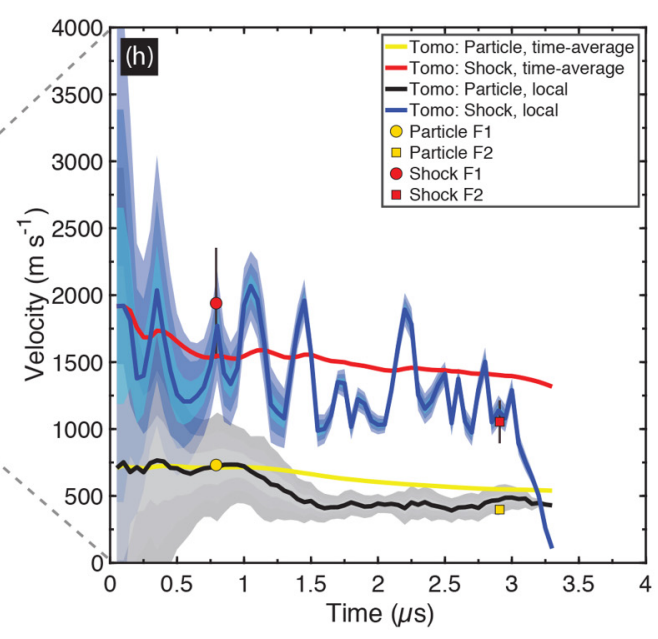

FIG. 4. Comparison of 2D simulations of shock propagation in chondrite precursor mixtures when the soda-lime sphere positions were randomly initialized [top row, (a)-(c)] and imported from vertical slices through the powder cell as measured with pre-shot X-ray tomography [bottom row, (d)-(f)]. (a) and (d) show the initial powder beds as porosity maps with the colors showing porosity according to the color bar below the figure. Porosity maps at the time of the experimental radiographs (relative to when the shock entered the bed) are shown in (b)-(c) and (e)-(f) for the randomly initialized and tomography-initialized cases, respectively. White lines mark the interface between the driver/powder interface (from which particle velocity is calculated, leftmost) and shockfront (rightmost), respectively. Local and time-averaged shock and particle velocities are shown in $(\mathrm{g})$ and $(\mathrm{h})$ for the randomly initialized and tomography-initialized simulations, respectively. Spatially averaged shock and particle velocities are shown as solid blue and black lines, respectively. One, two, and three standard deviations about these means are shown as blue and gray bands, respectively. The velocity data (red and yellow markers) represent the mean of the velocities in the on-axis regions, which spanned the vertical extent of the vertical, black arrows. Three standard deviations in particle velocity were $\pm 42 \mathrm{~ms}^{-1}(\mathrm{~F} 1)$ and $\pm 21 \mathrm{~ms}^{-1}(\mathrm{~F} 2)$, which are comparable to the marker sizes.

Table I. Figures 4(a) and 4(d) show the initial microstructures used in the simulations. In comparison with the randomly generated microstructure, the experimental microstructure is less uniform with a heterogeneous spatial distribution of soda-lime grains of varying size. Quantitatively, the nearestneighbor distances and two-point correlation functions in two simulation domains can be compared. The randomly initialized simulation bed had a mean nearest-neighbor-distance of $540 \mu \mathrm{m} \pm 20 \%$, whereas the tomography-initialized simulations had a smaller mean nearest-neighbor-distance of $406 \mu \mathrm{m}$ with a larger standard deviation of $\pm 28 \%$. Moreover, the clustering seen in Fig. 4(d) is borne out in the two-point 
correlation analysis, with almost twice (1.93-fold) the probability of soda-lime spheres being separated by one grain diameter than in the random case, for example. It is acknowledged that some of the grain size heterogeneity in the experimental microstructure has arisen due to extracting one slice from the tomographic volume, but the scope of this work is limited to comparing the idealized and single slice cases, which will likely provide a lower bound on the capabilities of artificially generated microstructures.

\section{Simulated velocity profiles and distributions}

From these two microstructures, iSALE simulations produced the porosity maps shown in Figs. 4(b) and 4(c) and Figs. 4(e) and 4(f) at the time of the experimental radiographs. Times are relative to the shock entering the powder bed. From the porosity maps, shockwave and driver positions, transit distances, and thus velocities were calculated in a time-averaged and local sense as described previously. Spatial averaging was performed over the on-axis regions analogously to experiment. These calculated velocity profiles are shown in Figs. 4(g) and 4(h) for the randomly initialized and tomography-initialized cases, respectively. Velocity profiles for tomography-initialized and randomly initialized simulations for all 7 shots are shown in the supplementary material. The mean, local shockwave, and particle velocities are shown as solid, dark blue, and black lines, respectively. Colored blue and gray bands show 1, 2, and 3 standard deviations from the mean about these local velocities. Overlaid are the timeaveraged shockwave and particle velocities, shown as solid red and yellow lines, respectively. Finally, experimental data are shown as solid red (shock) and solid yellow (particle) markers with error bars representing \pm 3 standard deviations; temporal uncertainties are too small to be seen on this scale.

Consider first Fig. 4(g). The local shockwave velocity exhibits large, quasi-periodic oscillations, which are attributed to the shockwave velocity increasing when inside a soda-lime microsphere and periodically slowing when inside the porous matrix. The excursions in local velocity about the time-averaged mean are on the order of 50-100\% and at early times, just as the shockwave enters the powder bed, extend over an even larger range. The modeled velocity profiles agree with the F2 shock and particle velocities within the error bounds and reproduce the global trend from high to low velocity. However, neither F1 velocities are reproduced within error. The lack of F1 velocity agreement supports the hypothesis that the high experimental velocities were due to a higher than intended soda-lime volume fraction near the driver, unaccounted for by the idealized simulation. The velocity profiles presented in the supplementary material show that the small grain mixtures exhibited more periodic velocity oscillations than that of the large grain mixtures, which was thought to be a result of the increased number of grains per powder bed.

Significantly, better agreement between the simulated velocity profiles and the early time experimental data is seen in the velocity profile extracted from the tomography-initialized simulation shown in Fig. 4(h). The particle velocity is notably $\sim 250-500 \mathrm{~ms}^{-1}$ faster at early times $(<1 \mu \mathrm{s})$ and is in excellent agreement with the F1 datum. The particle velocity now also exhibits significantly more temporal variation in magnitude than the randomly initialized case. While the oscillations in shock velocity about the mean are similar to the randomly initialized simulation, the time-averaged velocity is larger, $\sim 1500 \mathrm{~ms}^{-1}$ vs. $1000 \mathrm{~ms}^{-1}$. Now, there is excellent agreement of the simulated shock velocity with the F1 datum. Moreover, all 3 pairs of outlier F1 shock and particle velocity data were predicted by 7 of the 9 (three slices for each shot) tomography-initialized simulations. In contrast, only 1 outlier velocity (Shot 6, particle velocity) was predicted by the idealized simulations. These results confirm that accounting for the real experimental microstructure is crucial to accurately modeling local behaviors in mesoscale modeling. For each of the 7 shots, there was some variation between simulated velocity profiles as a function of angle about the vertical axis but no significant variation in the agreement with experimental data. Future work should repeat these experiments with more than two radiographic measurements per experiment in order to further understand the sensitivity of the mesoscale simulations to choice of tomography slice. Such an experiment would be an ideal opportunity to exploit the newly developed three-camera system reported by Escauriza et al. ${ }^{35}$

\section{Predicted data scatter and dispersion}

Despite the precisely controlled impact velocities in the experiment, the velocity data in both F1 and F2 were scattered over shock velocity-particle velocity space (Figs. 2 and 3). While the continuum-level predictions in Fig. 3 suggest that spatially averaged variations in sample composition can increase the range over which velocity data are measured, Fig. 5 shows that mesoscale analysis is required to approach a more accurate understanding of the experimental data scatter. In Fig. 5, experimental velocity data for both F1 and F2 are shown as filled black markers. Velocity data extracted from the mesoscale simulations in an analogous manner to the experimental measurements are shown as white markers. In F2, distances and times were taken relative to F1, further reducing uncertainty associated with the impact-to-F1 times. The simulated velocities reproduced the majority of scatter in the measured particle velocities although the simulations did not predict the particle velocity data observed below $400 \mathrm{~ms}^{-1}$. This is understood to be primarily a result of the simulations not reproducing the full experimental geometry and release conditions, which would have acted to slow the shock and particle velocities over time.

In general, the extremities and scatter of velocity data are not captured by the time-averaged calculations from the mesoscale models but are in some cases by the local calculations, with better agreement when the real experimental microstructure is used. To more accurately reproduce experimental velocity measurements, the inherently 3D nature of the shock propagation process, ${ }^{8}$ the radiographic diagnostic and tomography measurements must be accounted for in the 


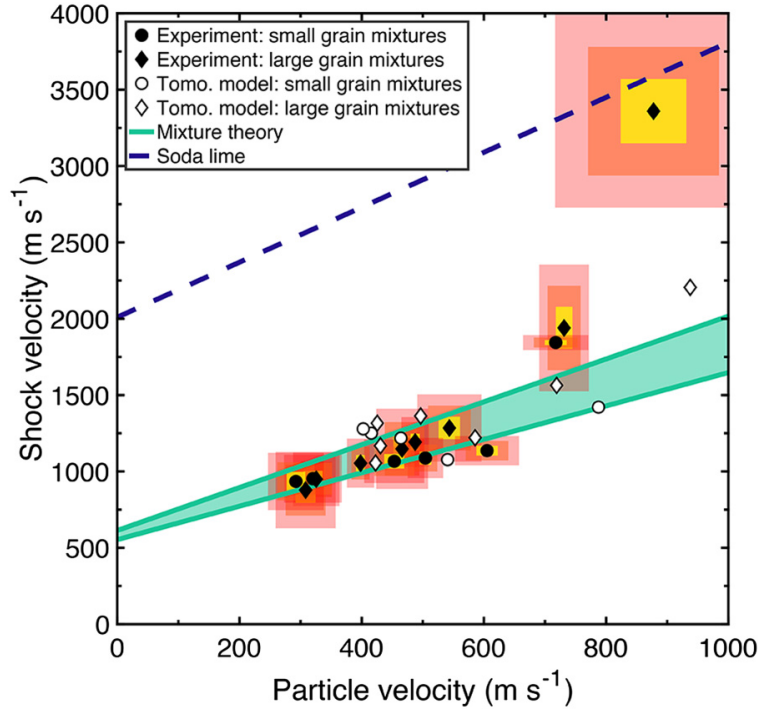

FIG. 5. All average velocity data recorded during the dynamic compaction of chondrite precursor mixtures. Each datum is shown with 1 (yellow), 2 (orange), and 3 (red) concentric rectangular bands representing 1,2 , and 3 standard deviations of the measured on-axis distributions. A band of green states shows the predicted velocity data for the measured on-axis volume fractions using X-ray tomography. A solid, blue, dashed line shows the solid soda-lime Hugoniot. Solid white markers show the velocity data calculated from tomography-initialized mesoscale simulations.

simulations. Experimental measurements were extracted from the central portion of radiographs as observed in a projection. These projections therefore sampled a cylindrical segment through the center of the powder volume and as a result were sensitive to a combination of both the soda-lime volume fraction in the central, on-axis column and outer portions of the powder cell. In contrast, analysis of mesoscale simulations was performed from the on-axis region in a strictly 2D simulation that did not account for the full powder cell geometry. Future investigations using pre-shot tomography should therefore include orientation markers to allow radiographic measurements to be more accurately reconciled with microstructural data. To investigate the sensitivity of the mesoscale model to variations in local fused silica density, a higher resolution tomography scan would also be beneficial. Alternatively, the gap between the idealized simulations and reality could be closed by defining the initial, idealized microstructures from 2D slices from an artificially generated 3D volume. This approach would go some way to reproducing the soda-lime particle size and shape distribution observed in Fig. 4(d).

The literature contains numerous reports of the lowimpedance component in a granular mixture dominating many aspects of the shock response such as dispersion and stress-strain-rate scaling. . $^{3,10,18}$ To further explore these conclusions in the context of this work, the mean shockwave velocities as a function of time were plotted for the soda-lime microspheres and porous fused silica matrix material separately. Figure 6 shows time-resolved, mean, local, and timeaveraged shock velocities in the on-axis region for the sodalime and matrix components for Shot 5. The downward trend in shockwave velocity over the duration of the experiment observed in Fig. 4(f) is exhibited in both the time-averaged and local matrix velocities. As expected, the time-averaged soda-lime velocity also follows this trend. The local soda-lime velocities, however, act to impose high-frequency oscillations on top of the time-averaged response. These observations are in line with previous analyses, which concluded that the matrix was responsible for a common deceleration of shockwave velocity across all 7 experiments in spite of varying impact stress and grain sizes. ${ }^{18}$

\section{E. Predicted temperature-pressure states}

Finally, the differences in simulated temperature and pressure in the powders were compared between the randomly initialized and tomography-initialized simulations in order to investigate the extent to which the non-uniformity of the sodalime grains influenced the local high-pressure-temperature $(\mathrm{P}-\mathrm{T})$ conditions.

Figure 7 shows pressure and temperature as a function of time extracted from simulations performed on Shot 5. The

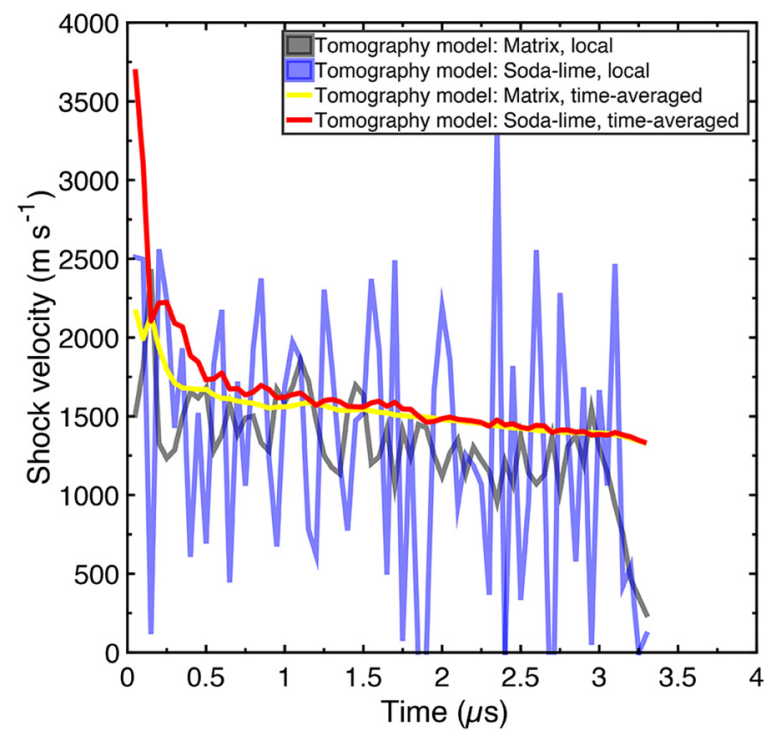

FIG. 6. Comparison of simulated shock velocity in the soda-lime and porous fused silica components for Shot 5 as a function of time. Solid red and yellow lines show, respectively, the time-averaged shock velocity inside the soda-lime and porous fused silica material, which are in close agreement as expected for stress transmission through the system. Translucent blue and gray lines show the local shock velocity in the soda-lime and fused silica components, respectively. In contrast to the local matrix velocity (gray), which is in close agreement with the time-averaged matrix velocity (yellow), the local soda-lime velocity (blue) dominates the velocity excursions observed in the mixture-averaged velocity data presented in Fig. 4. 


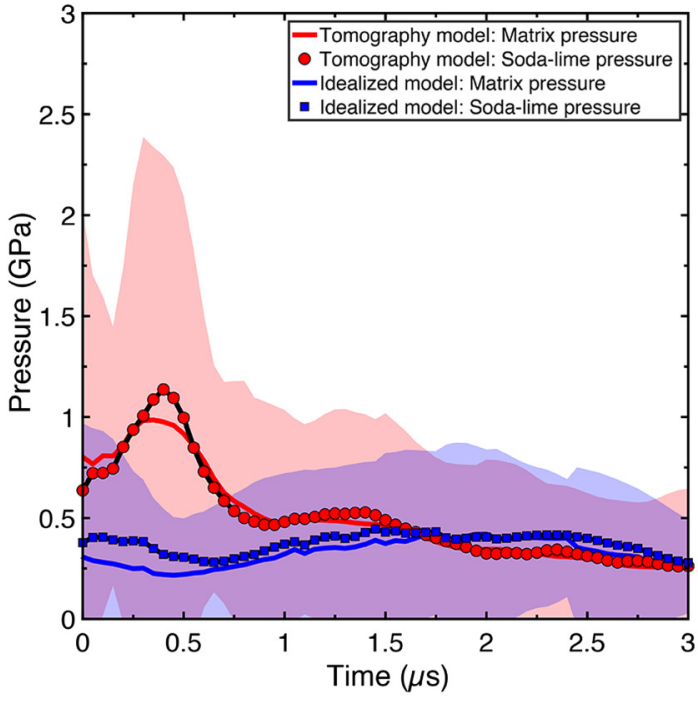

(a)

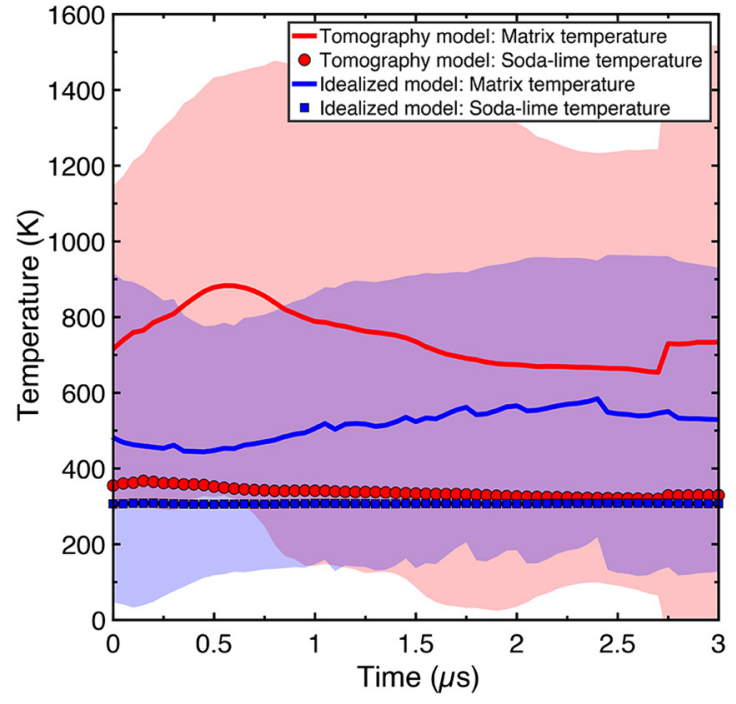

(b)

FIG. 7. Simulated pressure and temperature in the simulation domains as a function of shock transit time in the powder beds taken from randomly initialized (blue curves, markers, and blue bands) and tomography-initialized (red curves, markers, and bands) simulations on Shot 5. The curves represent the mean of the on-axis region. Colored bands, representing 3 standard deviations about the mean, are shown for the matrix material only.

temperature and pressure results presented are averages over the on-axis areas at a time after impact. As such, temperature and pressure are averaged over an increasingly large portion of the simulation domain as the shock front propagates. Temperatures were calculated with a constant heat capacity and the assumption that all heat generated during the compaction process goes into heating the matrix. Calculations were repeated for three tomography slices about the vertical axis. Figure 7(a) shows that the pressure difference between the soda-lime and matrix materials in both simulations is small as expected from continuity of pressure at interfaces during the compaction process. In contrast, Fig. 7(b) shows a large dichotomy in the predicted temperature between the soda-lime and matrix components for both simulations. Figures showing pressure and temperature as a function of time for all 3 experimental microstructures are shown in the supplementary material.

Across the 3 microstructures extracted from the tomography-initialized simulations [one of which is shown in Fig. 7(a)], significant excursions in mean matrix pressure toward $1 \mathrm{GPa}$ are seen for the first $\sim 0.5 \mu \mathrm{s}$. In contrast, the mean matrix pressure was comparably constant around $\sim 0.4$ GPa in the idealized simulations. At late times, the two simulations show similar pressure magnitudes. The larger pressures at early times in the tomography-initialized scenario are consistent with the higher volume fraction of soda-lime shown in Fig. 4(d) driving matrix material trapped between multiple grains to high pressures in a ring-up process. The temperature traces presented in Fig. 7(b) show that in the tomography-initialized simulations, the matrix experienced a mean temperature of $\sim 750 \mathrm{~K}$ in comparison with $\sim 500 \mathrm{~K}$ in the randomly initialized case.

The distribution of matrix pressures and temperatures, as judged by standard deviations about the mean, is also notably larger in the tomography-initialized case, which highlights the influence of grain arrangement on thermodynamic conditions in the system. Returning to the astrophysical impact-induced meteorite formation that motivated the earlier experiments, ${ }^{18}$ it is pertinent to comment on the relative likelihood of phase changes in the silica matrix as a result of the initial granular arrangements. Peak on-axis P-T conditions in the randomly initialized case are $\sim(0.41 \mathrm{GPa}, 460 \mathrm{~K})$, which extend up to $(0.93 \mathrm{GPa}, 850 \mathrm{~K})$ at three standard deviations from the mean. Even at this extreme, the matrix material lies within the $\alpha$-quartz region of silica phase space. ${ }^{36}$ In the tomography-initialized simulations, however, the distribution of on-axis P-T conditions extend from a peak of $\sim(1.2 \mathrm{GPa}$, $798 \mathrm{~K})$ to $(2.1 \mathrm{GPa}, 1385 \mathrm{~K})$. These more extreme conditions drive a fraction of the matrix material into the $\beta$-quartz region of phase space. Figure 9 in the supplementary material shows the two calculated P-T states plotted on the phasediagram for silica, taken from Gleason et al. ${ }^{36}$ This result shows clearly that initial granular arrangement can have a marked response on the thermodynamic conditions in a shocked bimodal powder system.

\section{CONCLUSIONS}

The influence of microstructure on the predicted distributions of shock velocity, particle velocity, temperature, and 
pressure in shock-compressed bimodal powder mixtures has been investigated. Quantitative component volume fractions measured with X-ray tomography prior to impact experiments were incorporated into mesoscale and continuumlevel models. By incorporating the real range of soda-lime volume fractions observed in the powder volume traversed by the shockwave, an improved agreement of continuum-level predictions with experimental velocity data was observed.

Real powder microstructures-extracted as 2D longitudinal sections from tomograms-were imported in a mesoscale shock physics code, and the results were compared with experimental data recorded on that same sample. The results of these tomography-initialized simulations were significantly different to those predicted by idealized simulations in which soda-lime microspheres were more uniformly distributed through the matrix volume. The mesoscale simulations showed quasi-periodic excursions in local shock velocity on the order of $50-100 \%$ about the mean and both simulation types predicted the majority of the experimental velocity measurements within error. However, only the tomographyinitialized simulations predicted the three outlier shock and particle velocities. This result confirms the importance of accounting for the real material microstructure and shows the dramatic effect non-uniform grain distributions can have on the measured shock response. Indeed, by separating out mean velocities in the matrix and soda-lime materials, it was shown that the time-dependent dispersion of the shockwave was limited by the matrix material with the soda-lime acting to impose high-frequency oscillations on top of this baseline. The unexpectedly high scatter of velocity data observed in the experiment was largely reproduced by the mesoscale simulations showing a strong influence of powder microstructure of shockwave and particle velocities despite otherwise consistent impact experiments and sample preparation methods.

Many different applications of dynamic granular compaction seek the ability to measure and predict the system's local thermodynamic state as a function of time in order to, for example, better tailor shock-driven material's synthesis mechanisms or understand meteorite microstructure. Understanding the distribution of high pressuretemperature states is a key step in this materials-by-design process. Here, a notable difference in the pressure-temperature states achieved in the compacted matrix was observed for the two powder microstructures, with the real microstructure experiencing larger, more varied temperature and pressure conditions. Simulations with a real microstructure predict the formation of a small volume of $\beta$-quartz during shock compression, whereas three standard deviations of the $\mathrm{P}-\mathrm{T}$ conditions in the randomly initialized simulation remain in the ambient $\alpha$-quartz phase. Future work that exploits the growing number of high-spatiotemporal, multi-frame imaging and diffraction methods under shock loading is clearly required to validate such predictions, but at this stage, it may be proposed that accurate, non-destructive powder packing characterization is a critical step in understanding the mesoscale shock response of granular systems.

\section{SUPPLEMENTARY MATERIAL}

See supplementary material for X-ray absorption calculations to examine the nature of the compaction waves, additional velocity data calculated from tomography-initialized mesoscale simulations, simulated temperature and pressure profiles for Shot 6 as a function of tomography slice angle about the longitudinal axis, and simulated T-P conditions on the silica phase diagram.

\section{ACKNOWLEDGMENTS}

The authors would like to acknowledge the technical staff at Imperial College London for preparing the sample components and ESRF for provision of the beamtime. D.E.E., D.J.C., and M.E.R. thank Imperial College London, AWE, and the University of Oxford for their support. M.E.R. also thanks Engineering and Physical Sciences Research Council (EPSRC) and AWE for providing doctoral and postdoctoral support through Grant Nos. EPSRC 1378728 and EP/R511742/1, respectively. Support for DEE provided by the Defence Science and Technology Laboratory (DSTL) is also gratefully acknowledged. We thank the iSALE developers (www.isale-code.de). J.G.D. gratefully acknowledges support from EPSRC EP/M506345/1. G.S.C. was supported by STFC (Grant No. ST/N000803/1). The authors also extend a sincere thank you to Philip Bland for stimulating work on the shock-driven meteorite formation and insight into our previous works. M.E.R. also thanks Alexander Rack (Beamline ID19, ESRF) for assistance with collection and reconstruction of the tomography data.

\section{REFERENCES}

${ }^{1}$ N. N. Thadhani et al., J. Appl. Phys. 82, 1113 (1997).

${ }^{2}$ D. E. Eakins and N. N. Thadhani, Int. Mater. Rev. 54, 181 (2013).

${ }^{3}$ P. A. Bland et al., Nat. Commun. 5, 5451 (2014).

${ }^{4}$ M. B. Syal, J. M. Owen, and P. L. Miller, Icarus 269, 50 (2016).

${ }^{5}$ C. D. Yarrington, R. R. Wixom, and D. L. Damm, J. Appl. Phys. 123, 105901 (2018).

${ }^{6}$ A. Britan et al., Shock Waves 23, 5 (2012).

${ }^{{ }^{7}}$ T. J. Vogler, M. Y. Lee, and D. E. Grady, Int. J. Solids Struct. 44, 636 (2007).

8. P. Borg and T. J. Vogler, Shock Waves 23, 153 (2012).

${ }^{\mathbf{9}}$ T. J. Vogler, C. S. Alexander, J. L. Wise, and S. T. Montgomery, J. Appl. Phys. 107, 043520 (2010).

${ }^{10}$ T. J. Vogler, J. P. Borg, and D. E. Grady, J. Appl. Phys. 112, 123507 (2012).

${ }^{11}$ J. LaJeunesse, S. T. Stewart, G. Kennedy, N. N. Thadhani, and J. P. Borg, "Investigating velocity spectra at the Hugoniot state of shock loaded heterogenous materials," in Shock Compression of Condensed Matter-2015: Proceedings of the Conference of the American Physical Society Topical Group on Shock Compression of Condensed Matter (AIP, 2017), p. 120007-6.

12J. W. LaJeunesse et al., J. Appl. Phys. 122, 015901 (2017).

${ }^{13}$ W. H. Holt, W. Mock, M. U. Anderson, G. T. Holman, and R. A. Graham, "Effect of particle morphology on input and propagated stress wave profiles for two highly porous polytetrafluoroethylene powders," in Shock Compression of Condensed Matter-1995: Proceedings of the Conference of the American Physical Society Topical Group on Shock Compression of Condensed Matter (AIP, 1996), pp. 573-576.

14J. L. Brown et al., "Dynamic compaction of sand," in Shock Compression of Condensed Matter-2007: Proceedings of the Conference of the American Physical Society Topical Group on Shock Compression of Condensed Matter (AIP, 2007), pp. 1363-1366. 
${ }^{15}$ D. E. Eakins and N. N. Thadhani, Acta Mater. 56, 1496 (2008).

${ }^{16}$ W. D. Neal, D. J. Chapman, and W. G. Proud, Eur. Phys. J. Appl. Phys. 57, 31001 (2012).

17J. P. Borg, W. R. Maines, and L. C. Chhabildas, J. Appl. Phys. 115, 213515 (2014).

${ }^{18}$ M. E. Rutherford et al., Sci. Rep. 7, 45206 (2017).

${ }^{19} \mathrm{H}$. Singh, Y. Mao, and A. Sreeranganathan, Model. Simul. Mater. Sci. Eng. 14, 351 (2006)

${ }^{\mathbf{2 0}} \mathrm{H}$. Singh et al., Comput. Mater. Sci. 44, 1050 (2009).

${ }^{21}$ M. E. Rutherford, "Studies of shock compression at the mesoscale with synchrotron X-ray phase-contrast radiography," Ph.D. thesis (Imperial College London, 2017).

${ }^{22}$ A. Lyckegaard, G. Johnson, and P. Tafforeau, Int. J. Tomogr. Stat. 18, 1 (2011).

${ }^{\mathbf{2 3}}$ N. Otsu, IEEE Trans. Syst. Man Cybern. 9, 62 (1979).

${ }^{24}$ D. Paganin, S. C. Mayo, T. E. Gureyev, P. R. Miller, and S. W. Wilkins, J. Microsc. 206, 33 (2002).
${ }^{25}$ S. S. Batsanov, Effects of Explosions on Materials: Modification and Synthesis Under High-Pressure Shock Compression (Springer, New York, NY, 1994), pp. 1-45.

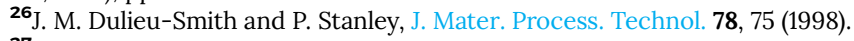
27. Wackerle, J. Appl. Phys. 33, 922 (1962).

${ }^{28} \mathrm{~K}$. Wünnemann, G. S. Collins, and H. J. Melosh, Icarus 180, 514 (2006).

${ }^{29}$ G. S. Collins, H. J. Melosh, and K. Wünnemann, Int. J. Impact Eng. 38, 434 (2011).

${ }^{30} \mathrm{D}$. J. Chapman, "Shock-compression of porous materials and diagnostic development," Ph.D. thesis (University of Cambridge, 2010).

${ }^{31}$ D. C. Drucker and W. Prager, Q. Appl. Math. 10, 157 (1952).

32J. E. Gorfain, C. T. Key, and C. S. Alexander, J. Dyn. Behav. Mater. 2, 83 (2016).

${ }^{33}$ G. S. Collins, H. J. Melosh, and B. A. Ivanov, Meteorit. Planet. Sci. 39, 217 (2004).

${ }^{34}$ J. G. Derrick, J. W. LaJeunesse, T. M. Davison, J. P. Borg, and G. S. Collins, Model. Simul. Mater. Sci. Eng. 26, 035009 (2018).

${ }^{35}$ E. M. Escauriza et al., Appl. Opt. 57, 5004 (2018).

${ }^{36}$ A. E. Gleason et al., Nat. Commun. 8, 1481 (2017). 